\section{From Monotherapy to Combination Therapies (CT): A Retrospective Review of the Process of Policy Transfer Among Four Sub-Saharan Countries (Uganda, Zambia, Sudan and Malawi)}

\section{Abstract}

Policy change in a dynamic environment amidst an increasing infectious disease caseload remains a herculean task. While it is ideal to drive the process using evidence, emergencies in such situation makes policy transfer the best option. However, considering the difference in contextual health system issues which could affect the policy performance, how does a 'wholesale' transferred policy perform in a changing environment? Who do international actors drive the process of policy transfer? What is the motivation of national actors to adopt a policy without context-specific evidence? Using the Dolowitz and Marsh model of policy transfer, this paper reviews the processes of policy change of malaria regimen from monotherapies such as chloroquine to artemisinin combination therapies. It assesses the fundamental influencers of this transfer and the strategies employed in making this change. Also, the challenges encountered during this process are discussed within the spectrum of the model. Lessons from this transfer process are relevant in guiding future policy changes of infectious diseases in other resourceconstrained settings in Africa.

Keywords: Policy transfer; malaria; Africa; infectious disease; resource-constrained setting

\section{Emmanuel Nene Odjidja ${ }^{1}$}

1 Institute of Global Health and Development, Queen Margaret University, Edinburgh UK

Corresponding author:

Emmanuel Nene Odjidja

\section{emmaodjidja@gmail.com}

Institute of Global Health and Development, Queen Margaret University, Edinburgh, UK.

Tel: +441314740000

\section{Citation: Odjidja EN (2017) From}

Monotherapy to Combination Therapies (CT): A Retrospective Review of the Process of Policy Transfer Among Four Sub-Saharan Countries (Uganda, Zambia, Sudan and Malawi). Health Syst Policy Res Vol. 4 No.4:61

Received: August 29, 2017, Accepted: September 28, 2017, Published: October 07, 2017

\section{Introduction}

The global health fight against malaria was threatened in 1972 when the first case of plasmodium falciparum (pf) resistance to chloroquine (CQ) and sulphadoxine pyrimethamine (SP) was reported [1]. These were the first line drug for treatment of uncomplicated malaria in Sudan, Uganda, Zambia and Malawi among many other countries experiencing high malarial morbidities [2-6]. The quest for answers amidst an increasing morbidity and mortality motivated Médecins Sans Frontières (MSF) to initiate in vivo studies with the aim of documenting the epidemiologic reach as well as finding a suitable alternative [7]. In April 2001, the World Health Organisation convened a technical consultation (in which MSF participated) in Geneva after which it strongly recommended artemisinin combination therapies as medicines for first line treatment of uncomplicated malaria [8] which was echoed in 2002 by the Roll Back Malaria Initiative [9]. As of 2013, 79 out of 88 malaria endemic countries changed their policy to adopt ACTs as a first line treatment drug [10], the majority occurred through a process of policy transfer [11]. Dolowitz and Marsh [12] define policy transfer as the process in which information on the development of policies, administrative arrangements and establishments is acquired from another setting that had previously used this information. Considering the procedure of decision making during the change process and documented evidence available in these countries, this paper will adopt the Dolowitz-Marsh Model of policy transfer [12]. Taking a retrospective insight into the processes of policy change, this paper will focus on answering the questions "why need for a transfer at the time, the actors involved, what was transferred in the process and how complete was it as well as the barriers encountered during and after these change processes" 
[12]. This will be a significant contribution to learning about the role of transfer in policy development because despite a change of antimalarial drug policy by several African countries, documentation on the processes has been limited [13]. A meticulous outlook of these change processes will therefore contribute to an appreciation of policy transfer and reveal the need for systematic lesson learning in health policy development. This paper will further argue that during the process of transfer, critical attention should be made towards contextualisation and local ownership as a way of naturally sustaining a policy and its implementation.

\section{Why the Need for a Policy Transfer Amidst Increasing Cases of Antimalarial Resistance?}

The first case of resistance to CQ had been reported in Brazzaville, Congo in 1985 and by 1999, multiple cases had been reported in several African countries that had contributed to rising levels of mortality and morbidity in most African countries [1]. For example, Sudan saw a dramatic rise of malaria deaths from 15000 to 35,000 in 1998 [14] and in Zambia, malaria cases had tripled from 121.5 cases/1000 people to 308 cases/1000 people between 1976 and 1999 which had further quadrupled by 2001 [15]. Meanwhile, in South East Asia (hitherto, witnessed resistance to CQ and SP), the efficacy of ART had been proven and widely used [16]. The global recognition of this problem and an available solution in South East Asia prompted global actors to adopt the idea of a policy transfer especially to sub-Saharan Africa. This swift but systematic process was necessary to impede the horrifying number of deaths across these countries and delay the extent of antimalarial resistance. As the WHO puts it "possible delay or slowing of spread of resistance to available effective and affordable antimalarial drugs, if included in combination therapy" [8].

\section{From South East Asia to Sub-Saharan Africa: Who were the Facilitators?}

In the process of policy change among countries, there is interplay of actors both locally and globally. Dolowitz and Marsh [12] classifies these actors into three main groups namely; insiders, outsiders and global actors. The antimalarial drug policy change in these countries gives a landmark understanding of how actors influence the processes of a policy change and the strategies employed to facilitate a transfer. This underpinning politics could further draw our insight into what and how agenda gets set for global health initiatives.

Insiders, according to Dolowitz and Marsh [12], are usually politicians and government officials who in the quest to solve a problem, learn from abroad and implement strategies or policies compatible with their local setting.

Health authorities in Zambia were taken by surprise by the unprecedented number of malaria cases which prompted them to seek advice from WHO leading to an adoption of Arthemeter Lumenfantre $(A L)$ in less than 3 months [4]. In this emergency, just like in most instances where insiders lead an idea for a policy transfer, local evidence plays a minor role in development processes [17]. Such abrupt actions of adopting policy change usually disallow contextualisation of initiatives and impede policy's ability to respond adequately to local needs. It also underscores what Hudson and Lowe [17] describe as the "absence of systematic lesson learning during transfer process". Authorities in Zambia again considered WHO's evidence is enough and credible [4] which clearly depicts the extent to which global evidence influences policy development processes in subSaharan African countries, especially during emergency public health situations.

MSF's strategic role in the change process of many African and Asian countries will be termed by Dolowitz and Marsh as an 'outsider' [12]. In the 1990s, although recognised by the world as an emerging problem, evidence to ascertain the reach and depth of $\mathrm{CQ}$ resistance were inadequate and scanty [7]. This inspired MSF to initiate two major studies with the aim of contributing to evidence on the epidemiologic extent. Following the initial studies, MSF went ahead to conduct 43 clinical trials in 18 countries out of which $83 \%$ were in Africa, with ten major studies conducted in Uganda and Sudan [7]. As of 2004, MSF had published 31 of its 43 studies in peer-reviewed journals, accounting for $23 \%$ of all malaria related articles [4]. This was followed with an evidencedriven advocacy for ACTs. For instance, in 2003, MSF published an article on the eve of WHO's report describing investments into other medicines apart from ACTs as "wasting money on funding drugs that don't work" [18]. Again, MSF participated in WHO's technical consultation on antimalarial drug combination therapy and advocated vehemently for the acceptance of ACTs using results from its studies [7]. These set of activities made MSF a key ambassador for the adoption of ACTs, as its recommendations were concordant with 11 countries' decision to consider a policy change including Sudan and Uganda [7]. In Sudan, MSF initiated and organised a milestone conference (treatment options against malaria in Sudan) jointly with WHO and the federal Ministry of Health Ministry (FMOH) that resulted in a recommendation of ACTs that same year [2]. In Zambia, MSF provided technical assistance to the ministry and further donated the country's first 800,000 AL tablets in February 2003 [4]. Uganda's joint taskforce on reviewing evidence of ACTs had MSF as a permanent representative [3].

While the approach of using evidence to influence policy change is laudable, generalising results based on non-representative geographic context could lead to making decisions based on partial knowledge and limited scope. As seen in Guthman's paper [7], MSF worked and conducted its research in politically unstable areas with fragile health systems; typical settings which had the potential of reducing malarial immunity of people and also increasing people's natural potency for any drug resistance. From this premise, it was obvious that MSF's campaign was based on limited scope and could have rather gained more plausible attention if directed towards calling for an increased research in different settings instead of a complete change in treatment medications globally.

The influence of global actions in the transition from CQ/SP to ACTs informed the dynamics of agenda setting among many 


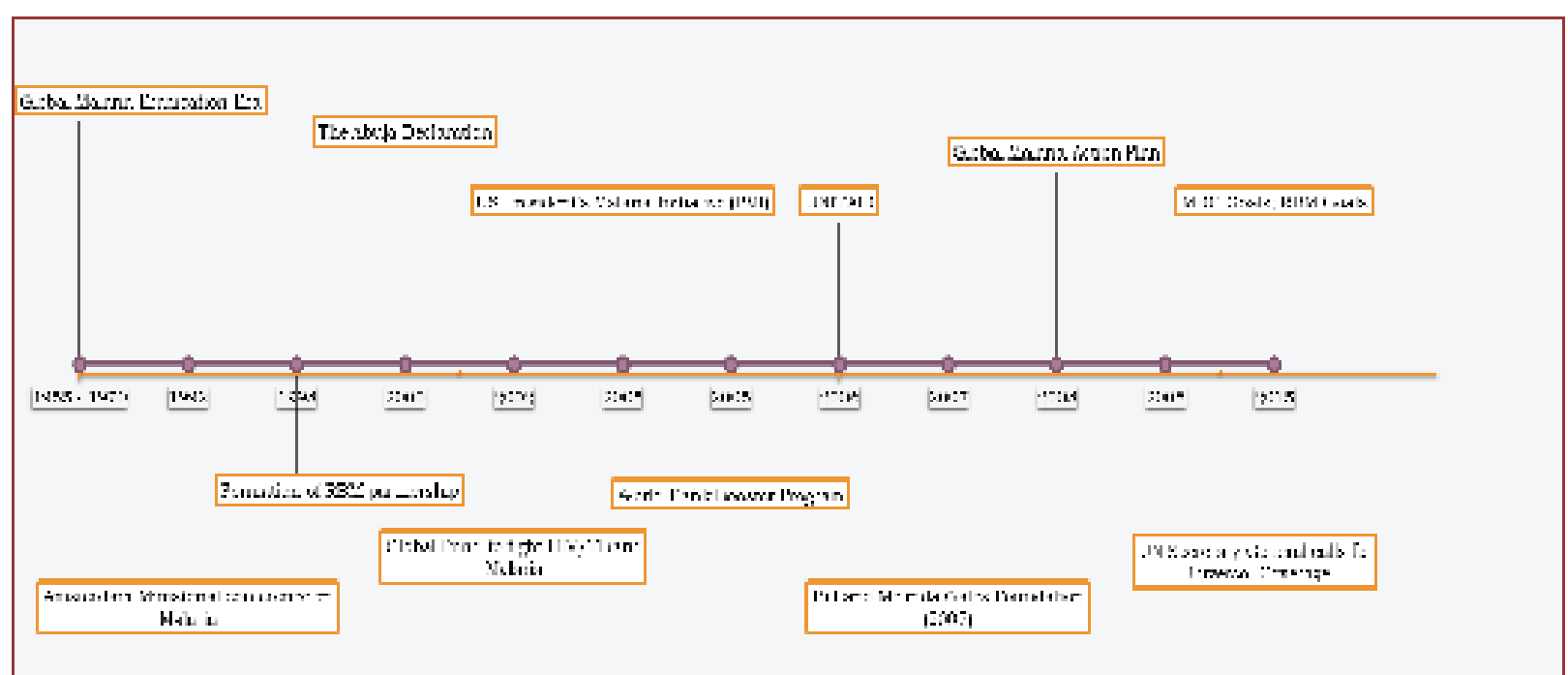

Figure 1

Timeline of global initiatives on Malaria and treatment initiatives

Source: RBM report, 2011

African countries [19]. Hudson and Lowe states that in order for WHO to improve health standards of people, it persuades nations to adopt the best practice, which is based on evidence from an acceptable epistemic body [17]. Since the malaria eradication era to date, WHO has encouraged supranational collaboration among global actors on the fight against malaria. Figure 1 shows a timeline of how WHO and other global initiatives have resulted on drawing global attention to discussions around CQ resistance and investments into ACTs.

In 1992, under the auspices of the executive board of WHO, health ministers were convened at a meeting in Amsterdam to discuss the malaria eradication strategy. This meeting attended by 44 ministers of health in 90 malaria endemic countries adopted the world declaration on control and treatment of Malaria. Key to resolutions reached was the recognition of $\mathrm{CQ}$ resistance and how that contributed to making treatment complicated. Participants (including ministers of health in Zambia, Uganda, Sudan and Malawi) therefore agreed to make this a priority, invest and engage in collaborative research to seek new and effective medicines [20].

The Roll Back Malaria (RBM) initiative was established by WHO and UNICEF with the aim of strengthening global campaign for increased resource allocation and research towards halving malaria deaths by 2010 [21]. This saw the commitment of World Bank and western countries pledge between $\$ 1.5$ billion and $\$ 2.5$ billion annually towards the fight against Malaria, Tuberculosis and HIV/AIDS [22]. In fulfilment of drawing political will towards the campaign, RBM conveyed a historic meeting of African leaders on April 2000 at Abuja [10]. This saw the birth of the Abuja Declaration where African leaders agreed to make $15 \%$ budgetary allocations to the health sector formulate individual national antimalarial drug policy and adopt the use of ACTs as medicine for first line treatment of malaria [10].

In acceptance to a proposal that emanated from the Abuja declaration, the Global Fund to fight HIV/AIDS, Malaria and
Tuberculosis (GFATM) was accepted by G8 countries and launched in 2002 as a private Swiss foundation by WHO with the aim of committing financial resources to low-resourced malaria endemic countries [23]. In 2003, RBM as a way of endorsing ACTs used GFATM's 30 million dollar aid (which was set up for the purchase of ACTs) as precursor for countries to change their antimalarial drug policy. Subsequently, more than 40 African countries adopted ACTs as first line treatment medicines and currently accessing aid from GFATM [24].

Between 2005 and 2007, other parallel global initiatives were launched namely; the US President's Malaria initiative (PMI), the World Bank booster program and Bill and Melinda Gates foundation initiative on Malaria [10]. These initiatives have financially supported countries with the purchase of ACTs and further delivered technical support for policy implementation.

\section{What was Transferred and How was it Completed?}

On realizing the effectiveness of Arthemeter Lumefantre (AL), WHO and Novartis signed a confidential memorandum of understanding which defined the terms by which Novartis would produce the cost and direct delivery to $\mathrm{WHO}$ [26]. Under the terms of engagement, WHO was responsible for distributing it to public sector authorities who agreed to join the program by using $A L$ as first line treatment medicine whiles African leaders under the GFATM program were responsible for making purchases directly through Global Fund under these special arrangements [25]. Zambia having encountered the highest number of cases in subSaharan Africa was the first to express interest to adopt AL [4]. In September 2002, authorities of the health ministry accepted and completely changed its policy to adopt AL as first line treatment while maintaining quinin for severe malaria treatment [4]. As at 2005, Sudan, Malawi and Uganda had fully changed their policies to adopt AL despite a higher price compared Artesunate 
Amodiaquine (AA), which was a suitable combination-based therapy for malaria treatment $[2,3,5]$.

\section{Challenges Encountered During and After Implementing}

Dolowitz and Marsh (2000) acknowledge that in the process of transfer, due to complexities that exist in different contexts, specific challenges might affect smooth implementation. The process of antimalarial drug change was not spared from challenges that affected and continue to threaten the future of ACTs in Africa. Economic worth of a country as cited by Hudson and Lowe [17] was a possible impediment to the adoption of policy change. In this case, all countries were economically poor with lower financial commitment to health. According to Sipilanyambe and his colleagues, ACTs cost 40 times more than CQ [4]. This made the governments of Uganda and Sudan initially reluctant to change its policy until it received funding from GFATM $[2,3,14]$. Despite changing its policy, Zambia initially encountered a huge deficit until it received initial donation of $800,000 \mathrm{AL}$ tablets from MSF [4]. Malawi faced a challenge of initial drug quantification, cost and a coherent understanding of drug administration [5]. There were challenges also around community access to ACTs because it was not delivered over the counter [5]. Again, as implied by Nanyunja and his colleagues, politicians' understanding of the evidence was inadequate which posed a challenge translating evidence into policy [3].

In spite of convincing evidence available in some countries on the inefficacy of $C Q$, there were doubts about the study results because of claims that donors played a major role in funding these studies. For example, one study carried out in Uganda's sentinel sites had been funded by WHO and considered as the most credible [26].

There was a clash of ulterior interests. For instance, in Kenya, private pharmaceutical companies stridently resisted the change of policy because there were huge quantities of SP and $C Q$ in stock during the period of intended change. Secondly, the sole sourcing mechanism employed by the WHO and GFATM meant that most local pharmaceutical companies will be out of business. The result; it took the Kenyan government 32 months to move into early implementation after public announcement [27].

Again, these countries were challenged with other health needs which were equally competing national interest, making prioritization of resources difficult. For example at the time of launching a new policy in 2002, Zambia had another conflicting national priority of launching an antiretroviral program in line with WHO's HIV strategy at the time. This resulted to difficulty in resource allocation [4]

\section{Reflections on the Process of Policy Transfer, Mechanisms Employed and the Dolowitz-Marsh Model}

The understanding of processes that encouraged policy transfer and influenced national prioritization of ACTs during antimalarial drug policy development gives a clearer picture of what Lowe and
Hudson [17] described as "mixture of consensual and coercive mechanism" in the adoption of policy change.

Given the economic status of these countries, it was obvious that the cost of implementation served as a disincentive to adopt ACTs. Nonetheless, the emergence of GFATM and other global donors facilitated global investments although its sustainability has been heavily questioned especially after the post GFATM era $[28,29]$.

Decision making on policy change processes were centered on a top-bottom approach with limited grassroots consultations. Clearly, this serves partly as a limitation to the Dolowitz and Marsh model because it fails to adequately establish a direction on how grassroots actors can instigate policy change. Actors involved in a policy transfer are hereby presented in a narrow spectrum of only insiders, outsiders and global players.

Another limitation in the Dolowitz and Marsh model of policy transfer is its failure to acknowledge the role of private sector play during a policy transfer process. Novartis funded some studies in Sudan and further provided 100,000 tablets [2]. These activities are not completely reflected in the process of transfer because the nature of this model allows limited understanding of how private sector either work independently or collaborate with government during policy transfer process.

Nabyonga-Orem and his colleagues suggested that the process of antimalarial drug policy change was a solely coercive one because GFATM insisted on funding only ACTs in some African especially Uganda [26]. This paper refutes this claim because at the time of considerations for a change in Uganda, three different research teams (two independently funded) had conducted quality research using the 'gold standard' (randomised controlled trials) and had consistent results on the efficacy of ACTs as against CQ and CQ/SP combined [30-32]. Such ground-breaking evidence gave GFATM a moral responsibility to have decided funding only ACTs.

\section{Conclusion}

Lessons from these countries have taught us that policy transfer can be complex especially with actors who have separate agendas and hidden motives. During the process, exhortations to drive political commitment should fervently include financial commitment. This is because as seen in these countries, GFATM still remains the major source of funding for ACTs yet 16 years after the Abuja declaration, African countries are struggling to allocate even 9\%. For instance in 2016, Malawi, Uganda, Zambia and South Sudan allocated just 7.98\%, 6.83\%, 8.30\% and $1.14 \%$ respectively to the health sector. Such unsatisfactory complimentary efforts connotes fewer commitments to overall health that obviously affects sustainability of funding streams of innovative health initiatives. Strong public private partnerships must be formed to strengthen funding sustainability and policy implementation.

Secondly, transfer of policy initiatives must be gradual, systematic and strategic. Given the huge imbalance in limited resources especially among African countries, there is a need to plan and target policy initiatives in a coordinated manner. Health 
authorities must seek the best evidence that will allow them to understand the context and target resources judiciously instead of abruptly adopting best practises from other settings.

Finally, another critical lesson here is the need to invest in surveillance, monitoring and evaluation. This is because, as

\section{References}

1 Trape J (2001) The public health impact of chloroquine resistance in Africa. The American J Trop Med and Hygiene 64: 12-17

2 Malik E, Mohamed T, Elmardi K, Mowien R, Elhassan A, et al. (2008) From chloroquine to Artemether-lumefantrine: The process of drug policy change in Zambia. Malaria J 5: 65.

3 Nanyunja M, Nabyonga Orem J, Kato F, Kaggwa M, Katureebe C, et al. (2011) Malaria treatment policy change and implementation: The case of Uganda. Malaria Research and Treatment. pp: 1-14.

4 Sipilanyambe N, Simon JL, Chanda P, Olumese P, Snow RW, et al. (2008) From chloroquine to artemether-lumefantrine: The process of drug policy change in Zambia. Malaria J 7: 25.

5 Malenga G, Wirima J, Kazembe $P$, Nyasulu $Y$, Mbvundula $M$, et al. (2009) Developing national treatment policy for falciparum malaria in Africa: Malawi experience. Transactions of the Royal Society of Tropical Medicine and Hygiene 103: S15-S18.

6 Mutero CM, Kramer RA, Paul C, Lesser A, Miranda M, et al. (2014) Factors influencing malaria control policy-making in Kenya, Uganda and Tanzania. Malaria J 13: 305.

7 Guthmann JP, Checchi F, Van Den Broek I, Balkan S, Van Herp M, et al. (2008) Assessing Antimalarial efficacy in a time of change to Artemisinin-Based combination therapies: The role of Médecins Sans Frontières. PLoS Medicine 5: e169.

8 World Health Organization (2001) Report of a technical consultation on antimalarial drug combinations. Geneva, Switzerland.

9 World Health Organisation (2007) Seminar notes on overview of malaria in Africa. Seminar series of the World Health Organisation.

10 World Health Organization (2014) World Malaria Report 2014.

11 Ngoasong MZ (2010) Transcalar networks for policy transfer and implementation: The case of global health policies for malaria and HIV/AIDS in Cameroon. Health Policy and Planning 26: 63-72.

12 Dolowitz DP, Marsh D (2000) Learning from abroad: The role of policy transfer in contemporary policy-making. Governance 13: 5-23.

13 Williams HA (2004) The process of changing national malaria treatment policy: Lessons from country-level studies. Health Policy and Planning 19: 356-370.

14 Malik EM, Khalafalla O (2004) Malaria in Sudan: Past, present and the future. Gezira Journal of Health Sciences 1: 9556-1728.

15 Chanda E, Kamuliwo M, Steketee RW, Macdonald MB, Babaniyi O, et al. (2013) An overview of the malaria control Programme in Zambia. ISRN Preventive Medicine pp. 1-8.

16 White NJ, Olliaro PL(1996) Strategies for the prevention of antimalarial drug resistance: Rationale for combination chemotherapy for malaria. Parasitology Today 12: 399-401.

17 Hudson J, Lowe S (2009) Understanding the policy process: Analysing welfare policy and practice (understanding welfare: Social issues, policy \& practice). [2nd ed] Bristol: Policy Press. experienced in most African countries especially Zambia, authorities were taken by surprise by the overwhelming number of $C Q$ resistance cases. To ameliorate this, health authorities must recognise the earnest need to invest into creating robust systems that report procedures of treatment and outcomes at the basic point of care.

18 Frankish H (2003) Money being wasted in fight against malaria, says MSF. The Lancet 361: 1530.

19 Newman RD, Moran AC, Kayentao K, Benga-De E, Yameogo M, et al. (2006) Prevention of malaria during pregnancy in West Africa: Policy change and the power of sub-regional action. Tropical Medicine \& Int Health 11: 462-469.

20 World Health Organisation (1992) Ministerial conference on malaria report by the Director-General. Geneva, Switzerland.

21 Nabarro DN (1998) Global health: The 'roll back malaria' campaign. Science 280: 2067-2068.

22 Narasimhan V, Attaran A, (2003) Malaria J 2: 8.

23 THE Global Fund to Fight HIV/Aids, Malaria and Tuberculosis (2005) History of the global fund - the global fund to fight AIDS, tuberculosis and malaria. The Global Fund to fight AIDS, Tuberculosis and Malaria.

24 Ogbonna A, UNEKE CJ (2008) Artemisinin-based combination therapy for uncomplicated malaria in sub-Saharan Africa: The efficacy, safety, resistance and policy implementation since Abuja 2000. Transactions of the Royal Society of Tropical Medicine and Hygiene. 102: 621-627.

25 Spar D, Delacey B (2004) The problem of Coartem In: Case studies of Malaria treatment. Boston: Harvard Business School Publishing, pp. 20-40.

26 Nabyonga-Orem J, Nanyunja M, Marchal B, Criel B, Sengooba $F_{\text {, }}$ et al. (2014) The roles and influence of actors in the uptake of evidence: The case of malaria treatment policy change in Uganda. Implementation Science Vol 9.

27 Amin AA, Zurovac D, Kangwana BB, Greenfield J, Otieno DN, et al. (2007) The challenges of changing national malaria drug policy to artemisinin-based combinations in Kenya. Malaria J 6: 72.

28 Shretta R, Omumbo J, Rapuoda B, Snow RW (2000) Using evidence to change antimalarial drug policy in Kenya. Tropical Medicine and Int Health 5: 755-764.

29 Kamya MR, Bakyaita NN, Talisuna AO, Were WM, Staedke SG, et al. (2002) Increasing antimalarial drug resistance in Uganda and revision of the national drug policy. Tropical Medicine and Int Health 7: 10311041.

30 Staedke SG, Kamya MR, Dorsey G, Gasasira A, Ndeezi G, et al. (2001) Amodiaquine, sulfadoxine/pyrimethamine and combination therapy for treatment of uncomplicated falciparum malaria in Kampala, Uganda: A randomised trial. The Lancet 358: 368-374

31 Dorsey G, Njama D, Kamya MR, Cattamanchi A, Kyabayinze D, et al. (2002) Sulfadoxine/pyrimethamine alone or with amodiaquine or artesunate for treatment of uncomplicated malaria: A longitudinal randomised trial. The Lancet 360: 2031-2038.

32 Ndyomugyenyi R, Magnussen P, Clarke S (2004) The efficacy of chloroquine, sulfadoxine-pyrimethamine and a combination of both for the treatment of uncomplicated plasmodium falciparum malaria in an area of low transmission in western Uganda. Tropical Medicine and Int Health 9: 47-52. 